\title{
The Role of Comfort and Discomfort in Insulin Therapy
}

\author{
Ronnie Aronson, M.D., FRCPC, FACE
}

\begin{abstract}
Despite the recognized importance of optimal insulin therapy, patient adherence to insulin therapy is an ongoing clinical care challenge. Insulin omission continues to be frequent and underestimated and has been correlated with poorer glycemic control and increased rates of diabetes-related complications. Insulin users consistently indentify multiple factors that contribute to insulin injection-related anxiety and to non-adherence. Injection-related discomfort continues to bear a significant contribution. Over the last decade, with advances in needle manufacturing technology, shorter and narrower needles have been associated with progressively improving patient self-rating of injection discomfort. Consequently, patient surveys of insulin users show discomfort to rank in the bottom third of significant contributors by prevalence. However, healthcare providers (HCP) and family member care providers continue to demonstrate a high level of anticipated and perceived pain for the patient. HCP anxiety and pain anticipation are each associated with patient anxiety and may therefore play a significant contributing role in patient non-adherence.
\end{abstract}

\section{Introduction}

S INCE THE 1922 INTROduction of insulin, patient self-care challenges have played a prominent role, leading, for example, to the early switch from intramuscular to subcutaneous injection. Awareness of intensive glycemic control grew significantly following the mid-1990s publication of the Diabetes Control and Complication Trial ${ }^{1}$ and the United Kingdom Prospective Diabetes Study. ${ }^{2}$ Their impact on clinical care standards was nearly immediate, effectively eliminating the then-common practice of dosing in response to hyperglycemia symptoms. The subsequent growth of evidence-based practice guidelines, which found a natural home in the complex and dynamic world of diabetes, further advanced the new imperative of adherence to intensive glycemic control.

Despite this recognized priority, adherence to insulin therapy has remained a clinical care challenge and is closely linked to poor patient outcomes. The DARTS Medicines Monitoring Unit ${ }^{3}$ found that glycosylated hemoglobin, diabetes complications, and diabetic ketoacidosis were related to omission of insulin. Twenty-five percent of this Tayside, Scotland, UK cohort were receiving less than two-thirds of their prescribed insulin. The Joslin Behavioural Research group found that $30.5 \%$ of their type 1 diabetes patients selfrestricted their insulin and that after a decade of follow-up, these patients had threefold higher mortality and a doubling in prevalence of nephropathy and foot problems. ${ }^{4}$ Fifty-seven percent of American insulin users reported omitting insulin that "they knew they should take." ${ }^{\prime 5}$ Similarly, the DAWN survey ${ }^{6}$ found that $20 \%$ of the respondents "often or sometimes" skipped their injections and 10\% restricted their number of daily injections. Insulin omission is also common internationally, varying from $19.9 \%$ in France to $42 \%$ in the United States and $44 \%$ in Japan. ${ }^{7}$ In a large managed care analysis of 27,000 type 2 diabetes patients newly started on insulin, prescription records identified that $4.5 \%$ did not fill their initial prescription, and $25.5 \%$ never refilled their first prescription. ${ }^{8}$

Anxiety plays a significant role in insulin therapy nonadherence. Although the DSM IV diagnosis of "blood-injectioninjury phobia" is as rare in diabetes populations (5\%) as in the general population (3\%), ${ }^{9,10}$ up to $94 \%$ of insulin-users did have symptoms of anxiety, distress, or phobias. ${ }^{10}$ The DAWN survey, ${ }^{6}$ for example, also found that 33\% "dreaded" their injections and $22 \%$ had to mentally prepare themselves for injections. Most important is that, in insulin-users, the presence of these anxiety symptoms was strongly associated with less self-monitoring, fewer daily insulin injections, ${ }^{11}$ poorer glycemic control, ${ }^{9,12}$ and a significant increased risk of cardiac and peripheral vascular disease. ${ }^{9}$

In insulin-refusers, anxiety, or unbased fear, may be even more common, reported by $61 \%$ of poorly controlled patients in an Israeli managed care setting, ${ }^{13} 29.5 \%$ of American patients "unwilling" to initiate insulin, ${ }^{14}$ and $48 \%$ of the TRIAD cohort of insulin-refusers. ${ }^{15}$ In newly diagnosed pediatric

LMC Diabetes \& Endocrinology, Toronto, Ontario, Canada. 
patients, $^{16}$ although $40 \%$ overall reported moderate-severe levels of fear, prevalence of fear reached $75 \%$ in children $<9$ years of age.

Anxiety similarly impacts compliance in other injectionusing populations, such as blood donors, ${ }^{17}$ pediatric venipuncture patients, ${ }^{18}$ and young adult travelers requiring vaccination. ${ }^{19}$ In multiple sclerosis patients, injection anxiety has also been found to be strongly associated with both nonadherence to disease-modifying therapies and with poorer outcomes. ${ }^{20}$

In surveys, patients have identified several contributors to injection anxiety, including poor healthcare provider (HCP) counseling $^{21}$ and their own limited health literacy, ${ }^{15}$ lack of self-confidence in ability to manage the injection logistics, ${ }^{8,22,23}$ and implication of disease severity. ${ }^{14,22}$

Injection discomfort is also consistently identified as a contributor to anxiety but with lower prevalence (from $8 \%{ }^{24}$ to $30 \%{ }^{15}$ ). Improvement in injection comfort represents a particular area of recent technological advance. However, even as comfort in insulin therapy has progressively improved for patients, it continues to be a contributor to injection anxiety, possibly to a greater extent among HCPs than among patients themselves.

We undertook a structured review of injection anxiety and the contributing role of pain or discomfort. Primary sources investigating injection pain and anxiety, published between 2000 and 2011, were collected from the Medline, Embase, Proquest, CINAHL, and Cochrane databases. "Injection," "pain," "needle," "comfort," "anxiety," "insulin," and "phobia" were used as key words. The searches were limited to the English language and human subjects, with publication types and age groups unselected to include a range of results. The reference lists of relevant articles were further reviewed to identify other publications where the key words appeared in the title. Articles were gathered by manual searches in Ovid Medline. To mitigate potential publication bias and maximize the search, doctoral theses and references listed in reviews and/or primary sources were also reviewed.

The database and manual searches yielded 94 publications. A personal collection of 40 additional sources included additional primary sources, posters, presentations, and a graduate thesis. Articles meeting the following selection criteria were included: (1) the study included approaches to control for confounding and other types of potential bias; (2) the full text of the article included a full description of the study design and methods used to measure and assess patientreported outcomes; (3) the publication was written in English or translated into English; and (4) study subjects were children, adolescents, and/or adults exposed to injection as a medical procedure.

\section{Injection Pain}

Patient awareness of injection discomfort has been studied extensively and is related to three key factors: needle length (and tissue level penetrated); needle diameter; and injection context. Injection context is defined by noise, view of the needle, and the apprehension of HCPs, both professional and family. Needles are typically manufactured by rolling a flat sheet of stainless steel or polymer into a tube, welding the seam, and then lengthening the resulting hollow tube over a mandrel core through an engineered dye. After cutting, the appropriate bevels, in the required angle, are then ground on one end, and a lubricating coating is applied to the chassis. Even as needle length and diameter are continually improved through advancing technology, a reproducible awareness of injection pain persists based on two environmental contributors: (1) the visual stimulus of the needle itself and (2) the level of pain anticipation among patients' HCPs.

\section{Needle length}

Modern insulin therapies are intended for introduction into the subcutaneous space. A needle needs to be long enough to successfully penetrate the dermis and short enough to avoid penetration of underlying sensitive muscle fascia, to avoid trauma and discomfort. Insulin absorption may also differ significantly between various sites. ${ }^{25}$ The highly innervated muscle fascia layer may account for many of the infrequent injection-related pain sensations (4.2-6.6\%), which are often described by patients as "I hit a nerve." ${ }^{21}$

Traditional $12.7 \mathrm{~mm}$ needles in children, despite appropriate skin-lift technique, have been found by sonography to result in an intramuscular location in $86 \%$ of injections. ${ }^{26}$ More recently, a computed tomography and magnetic resonance series $^{27}$ examined pediatric skin-bone thicknesses in midWestern American children to reduce overpenetration to bone of intramuscular vaccination needles. It is ironic that the $12.7 \mathrm{~mm}$ needle, long-used for subcutaneous injections, would not only overpenetrate skin but would also overpenetrate muscle and therefore injure the periosteum in $1.8 \%$ of deltoid injections. The comparable risk of overpenetration to bone may be even higher in children of developing countries.

Birkebaek et al. ${ }^{28}$ have since showed that in prepubertal children, up to $84 \%$ of girls and up to $95 \%$ of boys had abdominal skin thickness of $<8 \mathrm{~mm}$. Measurements at the thigh were far more variable depending on the site assessed, and measurements at the buttocks showed a generally higher skin thickness. It is important that skin compression during injection reduced skin thickness by up to a further 35\%. Needle lengths for subcutaneous injections should therefore not be longer than $8 \mathrm{~mm}$ and, arguably, not longer than $6 \mathrm{~mm}$.

Shorter needle lengths have also been consistently shown to not negatively affect glycemic control. Ross et al. ${ }^{29}$ first proved this point comparing $12.7 \mathrm{~mm}$ needles with $8 \mathrm{~mm}$ needles in 1999. Several subsequent investigations confirmed that with further decreasing needle lengths of $6 \mathrm{~mm}, 30,31$ $5 \mathrm{~mm},{ }^{32-35}$ and $4 \mathrm{~mm},{ }^{36,37}$ glycemic control remains unaffected. Mean scores from pain rating scales have generally also found a significant reduction in anticipation of pain ${ }^{38}$ and in injection pain with shorter needles, ${ }^{32,33}$ especially when assessed at home in relation to the injection itself. However, when blinded to needle length, patients find no overall difference in pain. ${ }^{31}$ Regardless, even if blinded users have not shown a difference in overall pain scoring, it remains possible that the discomfort of the occasional inadvertent intramuscular injection (the "I hit a nerve" sensation) may still be further reduced by shorter needles.

\section{Needle diameter}

In 1999, investigators at the Centre for Sensory-Motor Interaction in Denmark published a comprehensive controlled evaluation of the mechanics of invasive injury to human skin, using a needle insertion device that could be controlled for 
both speed and force of injection. ${ }^{39}$ By varying needle diameters and including a measure of skin distensibility, they were able to determine workforce of an injection and then correlate each of these measures to healthy subjects' injection pain. They identified that higher-gauge (narrower-diameter) needles are associated with less penetration force and lower injection workload, resulting in fewer reports of injection pain. They were also able to confirm the long-held suspicion that bleeding is more likely to be associated with injection pain and, in an important finding, that finer-gauge needles cause less bleeding and therefore less pain related to bleeding.

Injection pain was correlated with injection workload and was proven higher for injections at $45^{\circ}$ (vs. $90^{\circ}$ ) and was consistently higher for men. It is interesting that speed of insertion ( $2 \mathrm{~mm} / \mathrm{s}$ vs. $19 \mathrm{~mm} / \mathrm{s})$ did not affect pain frequency but did affect pain quality: slower injections were more likely to cause dull pain, whereas faster injection speeds were more associated with sharp pain.

Patients experience varying degrees of injection pain in different anatomical injection areas, although the cause of this variability had not been well understood. Using the same device to administer controlled injections, along with sonographic measures of tissue density, the same investigators subsequently showed no difference in pain frequency between anatomical regions. ${ }^{40}$ Penetration force declined from thigh to deltoid to abdomen, but because the thigh has lower skin distensibility, the overall workload for injection was found to be lower in the thigh than both the abdomen and deltoid area.

The most common type of pain described overall was sharp (67-76\%) versus dull. Dull pain is due mainly to C-fiber nociceptor activity, whereas sharp pain is mainly due to A-delta fiber activity. ${ }^{41}$ Because C-fibers are thought to respond to temporal summation of stimuli, slower injections, lasting nearly 10 times longer, would be more likely to activate Cfibers and initiate a dull pain sensation than a fast injection. Separately, pain associated with bleeding complications may be related to inflammatory mediators or may be directly related to stimulation of vascular nociceptors.

Injection pain is generally documented to diminish with the patient's injection experience, leveling off after 5 years of selfinjection. It is interesting that, under these controlled injection conditions, investigators could not find any sensitization or habituation.

Nearly all subsequent studies, blinded and unblinded, have found similar reductions in injection pain with increasing needle gauge. A subsequent study again using the same Danish controlled injection device went on to compare five different needle gauges, from 27-gauge to 32-gauge, in blinded healthy volunteers ${ }^{42}$ and confirmed the same pattern. Furthermore, for four of the needles, bleeding complications were also correlated with needle diameter, and bleeding events were associated with higher pain scores. More recently, a similar study examining response in 30-gauge versus 29-gauge versus 27-gauge syringe needles also showed that penetration force correlated with needle diameter. ${ }^{43}$ Both patients and HCPs have reported less pain and greater preference for thinner needles in comparisons of 33-gauge tips versus 31-gauge needles (unblinded), ${ }^{44}$ 29-gauge versus 27-gauge in blinded multiple sclerosis patients using prefilled syringes, ${ }^{45}$ 29-gauge versus 27 -gauge in blinded multiple sclerosis patients, ${ }^{46} 32$ gauge versus 30-gauge pen needles (unblinded), ${ }^{47}$ 32-gauge versus 31-gauge pen needles, ${ }^{38}$ and in comparison of 31-gauge needles, normal to thin wall. ${ }^{48}$ Finally, Japanese patients found less pain and bruising using a 33-gauge needle in a crossover study with a 31-gauge comparator needle. ${ }^{49}$

One small study of 12 patients in Germany ${ }^{50}$ was unable to document a difference between 27- and 28-gauge needles, although the study was underpowered.

In contrast, two well-designed studies were not able to confirm a relationship between comfort and needle gauge. In two sufficiently powered studies of 15 -year-olds with a mean diabetes duration of 6 years, Hanas et al. ${ }^{51}$ found that patients could not differentiate among three different gauges of needles (28-, 29-, and 30-gauge). Similarly, Schwartz et al. ${ }^{31}$ found no difference in Visual Analogue Scale scores among 15-year diabetes veterans comparing 31-gauge and 29-gauge needles. However, both populations studied were very experienced injectors and received their blinded injections by a designated nurse or were asked to recall their prior home experience at the study visit, rather than more optimally recording the home experience in a real-time diary. Injections provided by a third party are often associated with lower overall pain ratings ${ }^{52}$ and perhaps reduced the subjects' ability to differentiate between needle gauges. More important is that both studies reported on pain severity rather than the more commonly reported outcome of pain frequency.

Needle tips have also been compared in studies of comfort. Asakura et al. ${ }^{44}$ compared two different tip gauges and found less pain with a 29-gauge microtapered needle that slimmed to a 33-gauge tip. In two French trials, covering 241 patients, less pain was found with a five-bevel tip ${ }^{45}$ that happened to be on a narrower 29-gauge needle. The comparison by Mayer et al. ${ }^{43}$ of piercing forces of three needle gauges also included comparisons of five-bevel and three-bevel tips, but found that gauge was the primary determinant of cutting and piercing force. In a trial to assess benefit to penetration force, individuals on a panel of blinded nurses administering injections were each able to differentiate between a three-bevel and fivebevel tip and rated the five-bevel as requiring 25\% less penetration force. ${ }^{53}$ Patients also found the five-bevel less painful, but the difference did not reach statistical significance. Very recently, Hirsch et al. ${ }^{54}$ studied needles from several manufacturers in several gauges, ranging from 30-gauge to 32-gauge, to compare a new five-bevel needle tip to the standard three-bevel design. Penetration force during computer-controlled insertions using a human skin substitute was $23.7 \%$ less using a five-bevel tip versus matched needles with a three-bevel tip. In a series of paired, blinded injection comparisons, five-bevel tips were found to be non-inferior by insulin-taking patients. In continued home usage thereafter, when asked to evaluate needles with a new design, the patients significantly preferred the five-bevel tip on parameters such as ease of insertion, comfort, and preference. Finally, in a third round of comparison testing, during which subjects were then unblinded and oriented to the reduced penetration force benefits of the five-bevel needle, patients continued to significantly favor the five-bevel design in each of the same rating parameters.

\section{Injection context}

In fetal development, afferent sensory fibers emerge and eventually conduct sensory excitation (nociception) back to 
the dorsal root ganglia, contributing to a local reflex arc. With further development, sensory fibers ascend to the brain, and the same sensory excitation can be perceived as pain. For a given degree of nociception, the perception of pain is quite variable and determined by temperament, ${ }^{55}$ perceptual sensitivity, and prior negative painful experiences. ${ }^{56}$

Perception of pain and the distress related to pain are each separate but related factors in consideration of comfort in painful procedures. In children undergoing dental injection, those with higher baseline anxiety experienced nearly double the intensity of pain. ${ }^{57}$ It is significant that, although pain itself may diminish with exposure in pediatric populations, the associated emotional distress does not itself typically diminish with exposure. ${ }^{58}$ Pain and distress can each be independently modified by various psychological maneuvers, including placebo anesthesia, distraction, and procedural counseling. ${ }^{59}$ A Cochrane collaboration review ${ }^{60}$ of psychological interventions for needle-related pain and distress found, for example, that distraction, hypnosis, and cognitive behavioral therapy were successful in reducing pain, distress, or both. However, the reviewers highlight the commonly seen discrepancy in level of distress perceived by independent observer versus by self-report (in children). Similarly, a pediatric venipuncture series comparing combinations of these interventions ${ }^{61}$ found partial benefits of both placebo cream and topical anesthetic (EMLA; Astra Zeneca, London, UK) cream in self-report of pain, but limited (EMLA) or no (placebo) impact on the associated distress. Goodenough et al. ${ }^{18}$ found that placebo with the suggestion of benefit led to lower independently observed distress behavior in children, even when the patients did not perceive a benefit to their level of pain.

In seeking to understand injection pain or distress, the dissociation between HCP observation versus patient selfreport becomes very important. Advances in injection technology, such as pens, needles, and needle tip design, have led to reduced patient awareness of pain, even to the point of making it increasingly challenging to further differentiate new technologies. Patient ranking of discomfort as a barrier is no longer in the "top 5 " in lists of objections. ${ }^{13,15}$ In a large observational study of German teenagers, only $10.5 \%$ ranked pain as the most annoying aspect of their injections. ${ }^{22}$ In a large United Kingdom survey of insulin-using adults, only $6.6 \%$ reported "sometimes" having pain with injection. ${ }^{21}$

Yet among HCPs - either professional or family membersthe persistent perception of patient discomfort may actually present the greater barrier to effective injection therapy. Surveys of pediatricians and pediatric trainees in vaccination settings ${ }^{62}$ and of pharmacists involved in diabetes self-care ${ }^{63}$ show a very high perception of patient pain/distress, discordant with typical patient self-reports. Vaccination anxiety in the eyes of pediatricians ranged in score from 7.7 to 8.3 out of 10 and was lower in female HCPs and those with greater injection experience; pediatric self-reports of vaccination anxiety, by comparison, are typically in the score range of 2-3 out of 10 for intramuscular injection. ${ }^{62}$ In a large survey, ${ }^{63}$ Canadian pharmacists described a mean anticipated patient pain score of 4 out of 10, versus a patient actual mean of 1.3 out of 10. An anticipated fingertip lancing pain score of 3.6 out of 10 was reported by the pharmacists versus a patient selfreport mean of 1.6 out of 10. Finally, in two large Internet surveys of pain in insulin injection, ${ }^{64}$ one for patients and one for HCPs, significant differences in perception of patient pain were seen. Patients reported mean injection anxiety scores of 1.5 out of 10 versus a mean score of 2.8 provided by the HCPs. Pain itself was reported at 2.2 out of 10 for patients versus the highest score among primary care providers of 2.9. In a ranking of injection-related problems, HCPs rated injection anxiety as the most frequent problem, whereas patients themselves ranked it as lowest. Sixty-two percent of primary care providers described their patient as less than satisfied with their insulin injections, whereas only $27 \%$ of patients actually rated themselves as low. Very similarly, among primary care providers in a managed care setting in Israel, ${ }^{13}$ reasons for non-initiation of insulin in their uncontrolled patients were as follows: patient fear of hypoglycemia, stated by $79.7 \%$ (vs. $12 \%$ of patients); inability to cope with the pain of self-monitoring, $53.9 \%$ (vs. $5.4 \%$ of patients); and inability to cope with the pain of injection, $48.4 \%$ (vs. $12 \%$ of patients). Other reports have shown an equally concerning lack of awareness of their patients' injection anxiety, by being able to accurately identify only $50 \%$ of those with the problem. ${ }^{65}$

Similar impact of HCP apprehension on patient discomfort or distress is seen when family members are the HCPs or healthcare supporters. Although parents are generally considered more accurate predictors of their children's pain, ${ }^{66}$ among mothers of newly diagnosed children with diabetes, ${ }^{16}$ $30.4 \%$ rated their child's injection pain as moderate-severe, versus the reports of only $22.7 \%$ of children. Of experienced mothers, $13.6 \%$ continued to report distress with injections versus $9.5 \%$ of their children. Most important is that the mother's perception of their child's pain was highly correlated with that child's glycosylated hemoglobin level at 1 year. Mothers' potential influence on a child's pain and distress has been documented in other populations as well, including children receiving venipuncture ${ }^{67}$ and in infants receiving vaccinations. ${ }^{68}$

\section{Conclusions}

Despite decades of experience and a prominent awareness of the importance of effective insulin therapy for optimal longterm diabetes health, insulin therapy adherence has been less than ideal. The most common patient challenge is injection anxiety, with additional barriers in self-confidence and the impracticality of the lifestyle of those with diabetes.

Comfort in insulin therapy has contributed significantly to injection anxiety. Injection comfort, however, has greatly improved over the past decade because of advances in needle design and manufacture and of further insights into effective insulin delivery. Needle length has reduced from a traditional length of $12.7 \mathrm{~mm}$ to the current standard of $4 \mathrm{~mm}$, with unchanged efficacy, less unwanted tissue trauma, and greater confidence of accurate drug delivery to the subcutaneous space. Needle diameter has improved from 28-gauge to 32and 33-gauge, with thinner walls permitting unimpaired insulin flow. Needle tips have improved in design with increasing bevel cuts, accomplished with reduced penetration force, without loss of stability. All three achievements have led to consistent gains in patient comfort.

The contextual factors in insulin injection-higher HCP perception of patient distress or pain in contrast with patient self-reporting-now represent the greatest potential area of further improvement. HCPs and family members harbor an exaggerated, anachronistic view of discomfort as a continuing 
barrier to injection. Further education and insight into the current positive patient experience may help HCPs and family caregivers provide a more supportive context for insulin injection and allow further focus on the remaining barriers to effective insulin therapy.

\section{Acknowledgments}

I would like to thank Hilary Campbell for her expert assistance in the search of the literature. This work was supported by an unrestricted grant from Becton, Dickinson and Company.

\section{Author Disclosure Statement}

No competing financial interests exist.

\section{References}

1. The effect of intensive treatment of diabetes on the development and progression of long-term complications in insulindependent diabetes mellitus. The Diabetes Control and Complications Trial Research Group. N Engl J Med 1993; 329:977-986.

2. Intensive blood-glucose control with sulphonylureas or insulin compared with conventional treatment and risk of complications in patients with type 2 diabetes mellitus (UKPDS 33). UK Prospective Diabetes Study (UKPDS) Group. Lancet 1998;352:837-853. Erratum in Lancet 1999;354:602.

3. Morris AD, Boyle DI, McMahon AD, Greene SA, MacDonald TM, Newton RW: Adherence to insulin treatment, glycemic control, and ketoacidosis in insulin-dependent diabetes mellitus. Lancet 1997;350:1505-1510.

4. Goebel-Fabbri AE, Fikkan J, Franko DL, Pearson K, Anderson BJ, Weinger K: Insulin restriction and associated morbidity and mortality in women with type 1 diabetes. Diabetes Care 2008;31:415-419.

5. Peyrot M, Rubin RR, Kruger DF, Travis LB: Correlates of insulin injection omission. Diabetes Care 2010;33:240-245.

6. Peyrot M, Rubin R, Lauritzen T, Skovlund SE, Snoeck FJ, Matthews DR, Landgraf R, Kleinebreil L: Resistance to insulin therapy among patients and providers: results of the cross-national Diabetes Attitudes, Wishes and Needs (DAWN) study. Diabetes Care 2005;28:2673-2679.

7. Barnett AH, Meneghini LF, Schumm-Draeger P-M, Peyrot M: The GAPP (Global Attitudes of Patients and Physicians in Insulin Therapy) study: identifying risk factors associated with injection omission/non-adherence in insulin treated patients with type 1 and type 2 diabetes. Diabet Med 2012;29 (Supp 1):168.

8. Karter AJ, Parker MM, Moffett HH, Ahmed AT, Schmittdiel JA, Selby JV: New prescription medication gaps: a comprehensive measure of adherence to new prescriptions. Health Serv Res 2009;44:1640-1661.

9. Bienvenu OJ, Eaton WW: The epidemiology of bloodinjection-injury phobia. Psychol Med 1998;28:1129-1136.

10. Berlin I, Bisserbe JC, Eiber R, Balssa N, Sachon C, Bosquet F, Grimaldi A: Phobic symptoms, particularly the fear of blood and injury, are associated with poor glycemic control in type 1 diabetic adults. Diabetes Care 1997;20:176-178.

11. Zambanini A, Newson RB, Maisey M, Feher MD: Injection related anxiety in insulin-treated diabetes. Diabetes Res Clin Pract 1999;46:239-246.

12. Hanas R, Ludvigsson J: Experience of pain from insulin injections and needle-phobia in young patients with IDDM. Practical Diabetes Int 1997;14:95-99.
13. Nakar S, Yitzhaki G, Rosenberg R, Vinker S: Transition to insulin in Type 2 diabetes: family physicians' misconceptions of patients' fears contribute to existing barriers. J Diabetes Complications 2007;21:220-226.

14. Polonsky W, Hajos TRS, Dain MP, Snoeck FJ: Are patients with type 2 diabetes reluctant to start insulin? Curr Med Res Opin 2011;27:1169-1174.

15. Karter JK, Subramanian U, Saha C, Crosson JC, Parker MM, Swain BE, Moffet HH, Marrero DG: Barriers to insulin initiation: the Translating Research into Action for Diabetes Insulin Starts Project. Diabetes Care 2010;33:733-735.

16. Howe CJ, Ratcliffe SJ, Tuttle A, Dougherty S, Lipman TH: Needle anxiety in children with type 1 diabetes and their mothers. Am J Matern Child Nurs 2010;36:25-31.

17. Oswalt RM: A review of blood donor motivation and recruitment. Transfusion 1977;17:123-135.

18. Goodenough B, Kampel L, Champion GD, Laubreaux L, Nicholas MK, Ziegler JB, McInerney M: An investigation of the placebo effect and age-related factors in the report of needle pain from venipuncture in children. Pain 1997;72: 383-391.

19. Nir Y, Paz A, Sabo E, Potasman I: Fear of injections in young adults: prevalence and associations. Am J Trop Med Hyg 2003;68:341-344.

20. Turner AP, Williams RM, Sloan AP, Haselkorn JK: Injection anxiety remains a long-term barrier to medication adherence in multiple sclerosis. Rehabil Psychol 2009;54:116-121.

21. De Coninck C, Frid A, Gaspar R, Hicks D, Hirsch L, Kreugel G, Liersch J, Letondeur C, Sauvanet JP, Tubiana N, Strausse K: Results and analysis of the 2008-2009 Insulin Injection Technique Questionnaire survey. J Diabetes 2010;2:168-179.

22. Karges B, Muche R, Moritz M, Riegger I, Debatin KM, Heinze E, Wabitsch M, Karges W: Low discomfort and pain associated with intensified insulin therapy in children and adolescents. Diabetes Res Clin Pract 2008;80:96-101.

23. Polonsky WH, Fisher L, Guzman S, Villa-Caballero L, Edelman SV: Psychological insulin resistance in patients with type 2 diabetes. Diabetes Care 2005;28:2543-2545.

24. Karges B, Muche R, Riegger I, Moritz M, Heinze E, Debatin KM, Wabitsch M, Karges W: Injection of acidic or neutral insulin and pain: a single-centre, prospective, controlled, noninterventional study in pediatric patients with type 1 diabetes mellitus. Clin Ther 2006;28:2094-2101.

25. Gupta J, Felner EI, Prausnitz MR: Minimally invasive insulin delivery in subjects with type 1 diabetes using hollow microneedles. Diabetes Technol Ther 2009;11:329-337.

26. Tubiana-Rufi N, Belarbi N, Pasquier-Fediaevsky LD, Polak M, Kakou B, Leridon L, Hassan M, Czernichow P: Shorter needles $(8 \mathrm{~mm})$ reduce the risk of intramuscular injections in children with type 1 diabetes. Diabetes Care 1999;22:16211625.

27. Lippert WC, Wall EJ: Optimal intramuscular needlepenetration depth. Pediatrics 2008;122:e556-e563.

28. Birkebaek NH, Johansen A, Solvig J: Cutis/subcutis thickness at insulin injection sites and localization of simulated insulin boluses in children with type 1 diabetes mellitus: need for individualization of injection technique. Diabet Med 1998;15:965-971.

29. Ross SA, Jamal R, Leiter LA, Josse RG, Parkes JL, Qu S, Kerestan SP, Ginsberg BH: Evaluation of $8 \mathrm{~mm}$ insulin pen needles in people with type I and type 2 diabetes. Pract Diabetes Int 1999;16:145-148.

30. Hofman PL, Lawton SA, Peart JM, Holt JA, Jefferies CA, Robinson E, Cutfield WS: An angled insertion technique 
using $6 \mathrm{~mm}$ needles markedly reduces the risk of intramuscular injections in children and adolescents. Diabet Med 2007;24:1400-1405.

31. Schwartz S, Hassman D, Shelmet J, Sievers R, Weinstein R, Liang J, Lyness W: A multicenter, open-label, randomized, two-period crossover trial comparing glycemic control, satisfaction, and preference achieved with a 31 gauge $6 \mathrm{~mm}$ needle versus a 29 gauge $12.7 \mathrm{~mm}$ needle in obese patients with diabetes mellitus. Clin Ther 2004;26:16631678.

32. Strauss K, Hannet I, McGonigle J, Parkes JL, Ginsberg B, Jamal R, Frid A: Ultra-short $(5 \mathrm{~mm})$ insulin needles: trial results and clinical recommendations. Pract Diabetes Int 1999;16:218-222.

33. Kreugel G, Beijer HJM, Kerstens MN, ter Maaten JC, Sluiter WJ, Boot BS: Influence of needle size for subcutaneous administration on metabolic control and patient acceptance. Eur Diabetes Nursing 2007;4:1-5.

34. Hofman PL, Derraik JGB, Pinto TE, Tregurtha S, Faherty A, Peart JM, Drury PL, Robinson E, Tehranchi R, Donsmark M, Cutfield WS: Defining the ideal injection techniques when using $5 \mathrm{~mm}$ needles in children and adults. Diabetes Care 2010;33:1940-1944.

35. Kreugel G, Keers J, Kerstens M, Wolffenbuttel BHR: Randomized trial on the influence of the length of two insulin pen needles on glycemic control and patient preference in obese patients with diabetes. Diabetes Technol Ther 2011; 13:1-5.

36. Hirsch LJ, Gibney MA, Albanese J, Qu S, Kassler-Taub K: Comparative glycemic control, safety and patient ratings for a new $4 \mathrm{~mm} \times 32 \mathrm{G}$ insulin pen needle in adults with diabetes. Curr Med Res Opin 2010;26:1531-1541.

37. Birkebaek NH, Solvig J, Hansen B, Jorgensen C, Smedegaard J, Christiansen JS: A 4-mm needle reduces the risk of intramuscular injections without increasing backflow to skin surface in lean diabetic children and adults. Diabetes Care 2008;31:e65.

38. Iwanaga M, Kamoi K: Patient perceptions of injection pain and anxiety. Diabetes Technol Ther 2009;11:81-86.

39. Egekvist $H$, Bjerring $P$, Arendt-Nielson L: Pain and mechanical injury to human skin following needle insertions. Eur J Pain 1999;3:41-49.

40. Egekvist $\mathrm{H}$, Bjerring $\mathrm{P}$, Arendt-Nielson L: Regional variations in pain to controlled mechanical skin traumas from automatic needle insertions to ultrasonography. Skin Res Technol 1999;5:247-254.

41. Torebjork HE, Ochoa JL: Specific sensations evoked by activity in single identified sensory units in man. Acta Physiol Scand 1980;110:445-447.

42. Arendt-Nielson L, Egekvist H, Bjerring P: Pain following controlled cutaneous insertion of needles with different diameters. Somatosens Motor Res 2006;23:37-43.

43. Mayer G, Knappertz V: Needles: a comparison study. European Medical Device Technology. November 1, 2009. www.emdt.co.uk/article/needles-comparison-study (accessed April 8, 2012).

44. Asakura T, Seino H, Nunoi K, Hashimoto K, Mutou T, Yamazaki K, Kakutani M, Toraishi KI, Kitaoka M, Daikoku H, Sugiyama K, Narasaki K, Tsjui K, Ohnishi S, Oto K, Tsujimoto T, Nakano R: Usability of a microtapered needle (TN3305) for insulin treatment in Japanese patients with diabetes mellitus: a comparative clinical study with a standard thin wall needle. Diabetes Technol Ther 2006;8: $489-494$.
45. Jaber A, Bozzato GB, Vedrine L, Prais WA, Berube J, Laurent PE: A novel needle for subcutaneous injection of interferon beta-1a: effect on pain in volunteers and satisfaction in patients with multiple sclerosis. BMC Neurol 2008;8:38.

46. Oleen-Burkey M: Pain and force comparison of 27- and 29gauge needles [poster]. Presented at the Teamwork, Trends and Technology: New Solutions in Multiple Sclerosis Meeting, sponsored by The Consortium of Multiple Sclerosis Centers, Atlanta, GA, May 27, 2009.

47. McKay M, Compion G, Lytzen L: A comparison of insulin injection needles on patients' perceptions of pain, handling and acceptability. Diabetes Technol Ther 2009;11:195-201.

48. Siegmund T, Blankenfield H, Schumm-Drager P: Comparison of usability and patient preference for insulin pen needles produced with different production techniques. Diabetes Technol Ther 2009;11:523-528.

49. Miyakoshi M, Kamoi K, Iwanaga M, Hoshiyama A, Yamada A: Comparison of patient's preference, pain perception, and usability between Micro Fine Plus 31-gauge needle and microtapered NanoPass 33-gauge needle for insulin therapy. J Diabetes Sci Technol 2007;1:718-724.

50. Chantelau E, Lee DM, Hemmann DM, Zipfel U, Echterhoff S: What makes insulin injections so painful? $\mathrm{Br}$ Med J 1991;303:26-27.

51. Hanas R, Lytzen L, Ludvigsson J: Thinner needles do not influence injection pain, insulin leakage or bleeding in children and adolescents with type 1 diabetes. Pediatr Diabetes 2000;1:142-149.

52. Berteau C, Schwarzenbach F, Donazzolo Y, Latreille $M$, Berube J, Abry H, Cotten J, Feger C, Laurent PE: Evaluation of performance, safety, subject acceptance, and compliance of a disposable autoinjector for subcutaneous injections in healthy volunteers. Patient Prefer Adherence 2010;4: 379-388.

53. Vedrine L, Prais W, Laurent PE, Raynal-Olive C: Improving needle-point sharpness in prefillable syringes. Med Device Technol 2003;5:32-35.

54. Hirsch L, Gibney M, Berube J, Manocchio J: Impact of a modified needle tip geometry on penetration force as well as acceptability, preference, and perceived pain in subjects with diabetes. J Diabetes Sci Technol 2012;6:328-335.

55. Rocha EM, Prkachin KM, Beaumont SL, Hardy CL, Zumbo $\mathrm{BD}$ : Pain reactivity and somatisation in kindergarten-age children. J Pediatr Psychol 2003;28:47-57.

56. Walco GA: Needle pain in children: contextual factors. Pediatrics 2008;122 (Suppl 3):S125-S129.

57. Kuscu OO, Akyuz S: Is it the injection device or the anxiety experienced that causes pain during dental local anesthesia? Int J Paediatr Dent 2008;18:139-145.

58. Broome ME, Rehwaldt M, Fogg L: Relationships between cognitive behavioural techniques, temperament, observed distress and pain reports in children and adolescents during lumbar puncture. J Pediatr Nurs 1998;13:48-54.

59. Suls J, Wan CK: Effects of sensory and procedural information on coping with stressful medical procedures and pain: a meta-analysis. J Consult Clin Psychol 1989;57: 372-379.

60. Uman LS, Chambers CT, McGrath PJ, Kisely SR: Psychological intervention for needle-related procedural pain and distress in children and adolescents. Cochrane Database Syst Rev 2010;(11):CD005179.

61. Tak JH, van Bon WHJ: Pain- and distress-reducing interventions for venipuncture in children. Child Care Health Dev 2006;32:257-268. 
62. Brady KA, Avner JR, Khine H: Perception and attitude of providers towards pain and anxiety associated with pediatric vaccine injection. Clin Pediatr 2011;50:140-143.

63. Diamond S, Matok I: Pharmacists' anticipated pain compared to experienced pain associated with insulin pen injection and fingertip. Can J Diabetes 2011;35:282-286.

64. Rubin RR, Peyrot M, Kruger DF, Travis LB: Barriers to insulin injection therapy: patient and health care provider perspectives. Diabetes Educ 2009;35:1014-1022.

65. Simmons JH, McFann KK, Brown AC, Rewers A, Follansbee D, Temple-Trujillo RE, Klingensmith GJ: Reliability of the Diabetes Fear of Injecting and Self-testing Questionnaire in pediatric patients with type 1 diabetes. Diabetes Care 2007;30:987-988.

66. Schneider EM, LoBiondo-Wood G: Perceptions of procedural pain: parents, nurses, and children. Child Health Care 1992;21:157-162.
67. Fradet C, McGrath P, Kay J, Adams S, Luke B: A prospective survey of reactions to blood tests by children and adolescents. Pain 1990;40:53-60.

68. Piira T, Champion GD, Bustos T, Donnelly N, Lui K: Factors associated with infant pain response following an immunization injection. Early Hum Dev 2007;83:319-326.

Address correspondence to: Ronnie Aronson, M.D., FRCPC, FACE LMC Diabetes \& Endocrinology Suite 106 1929 Bayview Avenue Toronto, ON, Canada M4G 3E8

E-mail: ronnie.aronson@lmc.ca 